IIUL IISER

LUXEMBOURG InSIITUTE OF SOCIO-ECONOMIC RESEARCH

Dissemination of the survey methodology from a practical point of view 
LISER Working Papers are intended to make research findings available and stimulate comments and discussion. They have been approved for circulation but are to be considered preliminary. They have not been edited and have not been subject to any peer review.

The views expressed in this paper are those of the author(s) and do not necessarily reflect views of LISER. Errors and omissions are the sole responsibility of the author(s). 


\title{
Dissemination of the survey methodology from a practical point of view
}

\author{
María Guadarrama \\ Data Centre Luxembourg Institute of Socio-Economic Research (LISER) \\ Nermin Plavsic \\ Data Centre Luxembourg Institute of Socio-Economic Research (LISER)
}

February 2020

\begin{abstract}
When there is a project that requires data collection, the machinery of Data Center (DC) is set in motion. As any machine, we need good inputs to develop and handle good outputs. The main goal of this paper is to explain the methodological process of a survey in our department as well as the information needed to develop a survey. We follow the presentation given by Professor Yves Tillé on 30 May 2018 focusing on the steps of a survey. We give special attention on the conception of a survey, the dissociation between the survey and the research project and the information required to develop the "data collection". This document aims to give a practical perspective to the survey process.
\end{abstract}

Keywords: Data Centre; data collection; research project; survey project; methodology. 


\title{
Dissemination of the survey methodology from a practical point of view
}

\author{
María Guadarrama * \\ Nermin Plavsic ${ }^{\dagger}$
}

\begin{abstract}
When there is a project that requires data collection, the machinery of Data Center (DC) is set in motion. As any machine, we need good inputs to develop and handle good outputs. The main goal of this paper is to explain the methodological process of a survey in our department as well as the information needed to develop a survey. We follow the presentation given by Professor Yves Tillé on 30 May 2018 focusing on the steps of a survey. We give special attention on the conception of a survey, the dissociation between the survey and the research project and the information required to develop the "data collection". This document aims to give a practical perspective to the survey process.
\end{abstract}

Keywords: Data Centre; data collection; research project; survey project; methodology.

\section{Introduction}

The history of surveys reveals something paradoxical, lately surveys matter for their quantity (amount) and not for their quality, for instance: web surveys without random sampling (Lyberg \& Weisberg, 2016) or "selfselected" surveys, surveys on only one part of the population Lohr (2010). Results coming from these surveys should be taken with cautiousness, i.e., results may not be possible to extend to a population without severe problems of large biased and inefficient estimators. However, it is true that in official statistics some statistics must be produced (ex. survey of culture, children wellbeing, reform parental leave, household finance and consumption) therefore, the methodological issue must to be now to focus on the surveys quality, (Wolf et al., 2016; Lohr, 2010). The quality of the survey is translated in this document through the idea of survey

\footnotetext{
*María Guadarrama, Luxembourg Institute of Socio-Economic Research (LISER), 11, Porte des Sciences, Campus Belval L-4366 Esch-sur-Alzette, Luxembourg. Email: maria.guadarrama@liser.lu

${ }^{\dagger}$ Nermin Plavsic, Luxembourg Institute of Socio-Economic Research (LISER), 11, Porte des Sciences, Campus Belval L-4366 Esch-sur-Alzette, Luxembourg. Email: nermin.plavsic@liser.lu
} 
as a procedure, which follows a clear sequence (or steps) where no (random) jump from one step to other is allowed. Data Center department counts on a specialized team who may intervene in the whole procedure, not only on the definition of sampling frame, sampling and weighting or data management, but even before, at the practical conception of the question of interest, at the drafting of the questionnaire, at the construction of the final data base among others.

In this sense, LISER invited Professor Tillé from Institut de Statistique de l'Université de Neuchâtel (Switzerland) to give a lecture on survey sampling entitled Ten important ideas in the theory of survey sampling on 30 May 2018. The aim of the lecture was to highlight the basics of survey sampling from a theoretical point of view. The guideline was the dissociation between the two frameworks for the foundation of inference, the model-based and the designbased theory. In design-based theory, the units (to be surveyed) are selected randomly and the only source of randomness is the sample drawn. The inference is a function of the sampling design. On the other hand, model-based theory assumes a model given the sampling design then, parameters are estimated with the sample and a prediction is done for the non-observed units. However, for some indicators both theories can coincide. The presentation also stated the implications of not using weights, the most important one is related to having to possibly deal with large biased estimators. Later, the steps for creating a weighting model (system) where enunciated. Furthermore, Professor Tillé insisted on the wrong idea of the word "representativeness". This word is misleading in statistics and it can lead to confusion in survey sampling theory therefore, it is barely used in this field. In fact, if representativeness is used when drawing a sample with the same proportion as certain (and not all) characteristics as in the population, it may be not the best sampling design for the desired study (Tillé, 2019; Neyman, 1934).

In this document, we extend the presentation of Professor Tillé as a way to explain the process we follow in the Data Centre, and also, to translate the idea of "survey quality" when a survey project is proposed. The key question we have to ask ourselves when dealing with a survey project is: if I (we) could ask the whole population, what would I (we) want to estimate? Here, we have to clearly have in mind what we want is a characteristic of the population (ex. poverty rate at national level) and we are not interested in the values of each unit (ex. person) in the population (whether the person in the population is below the poverty line). We are also not interested in the values of the each unit (ex. person) in the sample as such, but on them as a tool to get the estimation of the characteristics of the population. Thus, we would like to underline the importance of having a clear notion of the whole survey project mainly: the objectives of the survey and the information needed (and available) -before the survey, during the survey, and after the survey. Once this is properly set, we can proceed with the design of the survey (as a whole process). We would like to insist on the idea of the survey quality. A proper survey may be obtained when proceeding after setting all information needed for the survey, and if and only if 
we proceed step by step without jumping the sequence. The consequences might be measured in terms of large bias of the estimators. This way to proceed is reflected in the template available in the Project Initiation Request (Déclaration d'intention de projet) at LISER.

We follow the steps of a survey enunciated in Professor Tillé' in the presentation: 1) Research of information on the topic of interest; 2) Definitions of the topics of interest; 3) Definition of the population and the sampling frame; 4) Construction of the sampling design; 5) Collection of the data; 6) Coding and creation of the data frame; 7) Estimation of the parameters. For this first document, we focus on the steps $1,2,3,4$, and 7 . Moreover, we consider it is important to distinguish between the research project and the survey project. We can contemplate this distinction as the step 0 of the survey procedure. The following sections of this document correspond to the steps of the survey mentioned above.

In this document, we cover only the methodological aspects of the survey process. We are preparing a document on best practices relating to questionnaire design, which will be helpful for adapting the survey questions to the research goals, improving the data quality, and reducing the burden for the respondents. There is a lot of literature on how to optimize questionnaire design. We will deal with key recommendations from theory and (our own) practice on topics such as question wording and question order, the cognitive efforts that respondents go through when answering a questionnaire, optimal rating scales, social desirability bias, and so on. We continue this document with our previous discussion on the various steps of a survey.

\section{Research information on the topic of interest}

Before we start examining the different steps in the survey process, it is important to first distinguish between a research project and a survey project. A survey is a tool or method which may allow us to answer (some of) the research question(s). In most cases, a survey is part of a research project but should not be viewed as the research project itself (meaning that a single survey by itself most probably will not address all issues raised within a research project). Additionally, surveys are best used to find partial explanations of a class of events, which means that they might not be an appropriate tool for the scope of the research undertaking (De Vaus, 2002). In that case it is advised to try another research method.

We strongly recommend starting the survey by establishing its aims. To do this, we need to review the relevant literature and look for existing information. This should drive the design of the questionnaire and help select relevant questions (Burgess, 2001). Brancato et al. (2006) points out that as soon as the "end-users" (end-users can refer to ministries, other researchers etc.) have announced their demand for data, we need to verify if information on the topic is already available.

In this phase, it is standard practice to collect literature on the issue, to 
search for published statistics, and to contact institutions which may have data (that are possibly still unpublished). Many questions dealing with demographic variables are common to many surveys, and some surveys, including original survey instruments, are available on the internet. However, this should not be misunderstood: for example, finding the wordings for demographic questions makes it easier to write them in a technically "good" way, but these questions still need to be asked in the new survey (they are a necessary information for the subsequent weighting process).

Thus, before formulating questions, we recommend starting with a review of relevant literature as well as analyses of data from other relevant surveys. If data already exists, you should use it as a matter of resource optimization. However, while taking advantage of the findings from the work of other researchers is good practice, we still need to verify all the questions, since findings from other surveys cannot guarantee that a question is appropriate in our new context (Fowler \& Cosenza, 2008).

Some questions to consider at this point:

- Do similar surveys exist?

- Were they tested? What were the results?

- Are there recommendations on the design?

- Is there a difference between our survey and previous surveys? Do we need another survey?

This first step should not be too costly nor time-consuming, yet it can help identify basic problems and structure the work. It should be done at the beginning of the questionnaire development before any draft is prepared. A helpful thing to remember at every step of the design process is that you should always know what you want answered, even if you already have the data.

This is where we get closer to the construction of the measurement instrument, the questionnaire. However, we are not there yet. A problem often encountered by survey practitioners is that many researchers make the mistake of asking too many questions Burgess (2001). We have to pay attention to the length of the questionnaire. Even more now that surveys are mostly web-based. We can see that the quality of surveys diminishes as they get longer and we recommend that ideally they take no longer than 20-30 minutes, especially web surveys.

The problems signaled above often arise from an incomplete analysis of how to meet the research and the survey goals. We should always strive to design concise questionnaires (this might also help get better response rates, reduce respondent burden, etc.). Always keep in mind: what type of question do we really want answered and, is a survey the most appropriate tool to answer our question? The research objectives and research questions should therefore be as clear as possible. This brings us to our next section. 


\section{Definition of the topics of interest}

After the definition of the basic elements of survey design, Brancato et al. (2006) recommend to transfer the general survey objectives into concrete research questions. This is the start of the conceptualisation phase. Often this important step is neglected as time constraints press researchers to draft a questionnaire before thinking in detail about the concept. However, to ignore such basics may result in unreliable and non-valid data, respondents' and/or interviewers' dissatisfaction, low nonresponse rates, etc. (Brancato et al., 2006).

That is why we insist on the fact that one of the most important tasks in a survey is to formulate the objectives. They establish not only the surveys broad information needs, but the operational definitions to be used, the specific topics to be addressed, what is to be included in the survey and what is to be excluded, and what information is really needed versus what would be nice to know (Franklin \& Walker, 2003). The design and method should always follow from your specific objectives.

A well-specified set of research objectives is such a critical component of the survey process because it will facilitate many of the decisions involved in survey design. Defining the research objectives is often accomplished best by identifying a small set of key research questions to be answered by the survey (Biemer \& Lyberg, 2003).

The time spent in the development of a set of research questions is time saved during questionnaire design since each research question can be linked to one or more data elements to be collected during fieldwork. It is good practice to ensure that every question on the questionnaire corresponds to at least one research question. You should ensure that the objectives of a research project are fully translated into a series of questions, which will then hopefully obtain the required data (Biemer \& Lyberg, 2003).

We conclude this section with a summary of the steps so far: initially, the survey will begin with general research issues that need to be investigated. These are narrowed down so that they become research questions. Once the research questions have been formulated, the planning of the fieldwork can begin (Bryman, 2016). Schwarz (1997) explains that the research objectives determine the concepts to be investigated. The concepts then need to be translated into appropriate questions. Starting from a set of research objectives, researchers furthermore specify the population of interest and draw an appropriate sample. The methodology used thus follows from the survey objective (which is based on the topic of interest). Adding further topics not related to the main one will make it difficult for us to ensure the suitability of the previously proposed methodology.

We have an example in the survey of Labor insertion of recent university graduates done by the Spanish National Institute in 2019: "Do you plan to move back to Spain?" When looking at the questions that come before it, this particular question "appears" to be in the proper context (mobility), but the 
question itself is still worded in a non-optimal way, and it is ambiguous and difficult to answer. This question lacks specification of temporary boundaries and it should be reformulated. We propose the following: "Do you plan to come back to Spain in the next 5 years?" (we can consider the 5 years as a post-doc or training period. The idea may be to get an indication whether the person plans to stay abroad or come back).

\section{Definition of the population and the sampling frame}

Once the topic(s) of interest is (are) set, the next step is the definition of the population of interest or the study population and the subsequent construction of the sampling frame. Both, the topic of interest and the study population must be clearly defined and related to each other, the methodology proposed would be set regarding that as main inputs. We should think for instance, whether topics related to specific population (workers, retired people, children) should be studied in a general population and if so, how. The population is, by definition, assumed to be discrete and composed by a finite number of elements (Tillé, 2019). The concept of population as the topic of interest, is an abstract concept that should be precisely defined as well as the survey objectives are translated into questions. The population may be defined with two criteria namely: unit of study and temporal dimension (Jann \& Hinz, 2016), we also add a third criteria, the geographical boundaries.

Normally, the unit of interest in social surveys is either individual (for instance in the culture survey or children wellbeing survey), households (Household finance and consumption survey -HFCS- or survey of income and living conditions -SILC-) or companies (survey on opening hours). The unit of interest may display certain features. For instance, individuals would be studied because they are workers or because they are elderly or children. On the other hand, firms can be studied because they belong to a specific activity for example factories, research companies or retail trade firms. The definition of the unit of interest should be clearly specified and it may not be exactly the same as the definition given by other stakeholders (the administration or even researchers from other field).

The temporal dimension or reference period distinguish the cross-sectional surveys from longitudinal studies or panel surveys. Cross-sectional surveys define a reference period or specific point in time in which the population is studied: fixed picture taken in a moment time. A longitudinal or panel survey is a survey that follows a population defined at time $t=0$ over a period of years $T$. This population (population at time $t=0$ ) is followed with the sample of respondents from $t=0$ that are updated each year $t(t=1, \cdots, T)$. In this case, the picture is taken on the same population but on different time periods. One of the uses of the survey may be to follow the evolution of that specific population.

The geographical characteristics set the location area of the study. Typically 
studies at LISER refer to units of analysis residing in the country of Luxembourg but they can also refer to other territories such as the Grand-Region or the crossborder countries. We would to highlight that the geographical limits of the study should be also accurately set and they do not necessarily coincide with definition stated by other research field/institution (for instance the administration). A clear example is the "the limits" of the Grand-Region that differ for different stakeholder. Therefore, it will be always necessary to precise the definition used at the level of the region.

The sampling frame is a list of all (available) units of the population defined with three criteria enounced above. Sampling frame can be also seen as the extended definition of the population (Tillé \& Matei, 2016). In practice, the sampling frame is built regarding the availability of data. The administrative registers are seen as the "more trustful" data sources for building a sampling frame (Lohr, 2010). In Luxembourg, there are two administrative registers: register held by the social security institute (in French IGSS) and the register of the physical persons held by the Centre de Technologie et d'Information de l'État (CTIE). Documentation of the register from IGSS is available online. The register held by IGSS is a fusion of several registers: the centre commun de la sécurité sociale (CCSS), the agence pour le développement de l'emploi (ADEM), the service national de la statistique (STATEC), the caisse nationale de santé (CNS), the caisse nationale d'assurance pension (CNAP), the fonds national de solidarité (FNS), the caisse pour l'avenir des enfants (CAE) and pension public schemes. In the documentation of the register, see IGSS (2019), it is set that This register contains a list of individuals who are linked to the national social protection system. As a consequence, international civil servants who are not affiliated to Luxembourg social security are excluded from the scope of the platform. The register CTIE contains a list of the persons who have a national identification number (13-digit number) in the Grand Duchy of Luxembourg (see the webpage of the Luxembourg office guichet.lu). There are other administrative registers such as the directory of firms in the national statistical institute of Luxembourg (STATEC) or the register of Luxembourgish schools held by the Ministry of Education, among others. We can think about other sources of data, telephone lists, but this can be considered as obsolete (as list for individuals) as not many people have land line and there is no (public) list of mobile phones.

Before building the sampling frame, it is important to understand the definition of the units given by the selected register. That means, for instance, that the IGSS does not allow the construction a sampling frame on the whole population living in Luxembourg but a register of workers enrolled in any of the social protection system of Luxembourg (no matter the place of residence). Moreover, IGSS register is a register of employers (the person that declares the workers), see IGSS (2019), and not a register of firms (no matter the definition of firms used). On the other hand, each register has their own definition that can or cannot fit with the survey interests. For instance, the mentioned register 
from STATEC gives a precise definition: A firm is an organizational unit for the production of goods or services with a certain degree of decision-making autonomy, in particular for the allocation of its current resources; a company carries out one or more activities in one or more locations. This definition is completed with the definition of a relationship of firm and legal unit, see STATEC (2018) for further details. Therefore the accuracy of the selected register should be analyzed to determine whether it covers the population under study.

In an ideal survey, the sampling frame covers the entire population under study, but this is seldom the case, and coverage problems do often come up. It is said that there is undercoverage when the units in the population are not in the sampling frame and overcoverage when the units in the sampling frame are not in the population. We can imagine a study on all people residing in Luxembourg at December 2018. There is no register that covers the whole population since homeless peole are not are not officially "registered". Moreover, with the availability of the registers in Luxembourg, it is not always possible to build a sampling frame that corresponds exactly to the population under study but a sort of "proxy" of the population of interest. Sometimes, the survey population (the population that will be sampled) does not coincide with the study population, for instance in HFCS, the unit of interest is the private household (see the methodological gidelines for the HFCS) and in Luxembourg, there is no register of private households but a register of fiscal households. In those cases, when the population of study does not correspond to the sampling frame, it is possible to use techniques that allow to compute the estimators in the population of interest with the data from the (population of the) sampling frame as the method of the Generalized Weighting Share Method (GWSM), explained in the book of Lavallée (2007).

Once the sampling frame is set, the sampling design will be run accordingly. At LISER, the sampling frame is typically unique but there is the possibility to obtain a sample (next step on the survey process) from multiple sampling frames. Further information in multiple sampling frames are available in Hartley (1974) or more recently Lohr (2007), or Lohr (2009).

\section{Construction of the sampling design}

\subsection{Framework}

Sampling design is one the most important steps in the survey process, in fact more important than the absolute value of the sample size. The pool done for American elections of 1936 by Literary Digest can be an example. They obtained a sample of 2.3 million people with which they predicted the victory of Landon with $55 \%$ but he received $37 \%$ of the votes versus the $61 \%$ for Roosevelt in the general elections. One of the problems of this survey was a coverage problem (Lohr, 2010) that was not solved by obtaining a large sample. In this section, we discuss how to construct the sampling design. 
In most of the cases, it is not possible to survey the whole population (here, we refer to population as the sampling frame), due to financial constrains or/and time (among other factors), then, we have to select a subset of the population for the data collection. The data obtained from this sub-population or sample will be used to make inference in the population. Therefore, there should be links between the sample and the population. These links will be obtained in a sort of random process that is the sample selection and which will take the form of the inclusion probabilities or sampling weights (the inverse of inclusion probabilities). Moreover, there is usually a gap between the sample draw and the respondent sample, which is called the non-response. In this section, we explain the sampling design, which takes into account both, the sample selection and the non-response adjustment. Even if the non-response adjustment is done after the data collection (end of the fieldwork) this procedure should be foreseen at the moment of the construction of the sampling design.

We need to introduce the concept of auxiliary information that will be crucial to develop the announced activities. Auxiliary information is defined as all external information that can improve the quality of the survey results, Tillé (2019). This information is referred to as the knowledge of the totals or the individual values of one (or several) variable(s) on the population. This information is (normally) available and it comes from the information associated to the sampling frame (associated to the units when defining the sampling frame). It also can come from a census. Auxiliary information may be used to obtain more precise results. This auxiliary information can be used at two levels, at the level of the conception of the sampling plan and at the level of the estimation of the parameters. At the level of the sampling plan, the auxiliary information is used to propose a sampling design that fits the objectives of the survey (Tillé \& Matei, 2016). At the level of the estimation, auxiliary variables are introduced in the calibration method to obtain accurate estimators with no need of specification of a model.

The auxiliary information available in Luxembourgish registers is the following. From the IGSS register, at the level of employees we find their: sociodemographic characteristics; characteristics of jobs; characteristics of employers; social benefits: family benefits, replacement benefits, social care; work absences; family relationships. At the level of employers, the information reveals their characteristics on the main activity sector, employer size and legal status. The register held by the CTIE contains a list of the residents (physical persons) in Luxembourg and their information on the surname and first name(s); national identification number; usual place of residence; date and place of birth; family status; nationality or nationalities, or statelessness; refugee or subsidiary protection status; gender; for those who are married, separated, widowed or in partnerships, the national identification numbers, surnames, given names and dates of birth of living or predeceased spouses; national identification numbers of parents where parentage has been established, provided that such numbers have been assigned; national identification numbers of children where parentage 
has been established, provided that such numbers have been assigned; origin of the data recorded and any modifications made; date and place of death; nobility titles for members of the royal family.

Regarding that, when the objective is related to income, it seems more convenient to use the IGSS database. Before moving forward, we also need to point out that if administrative registers are used, the possible legal restrictions and the administrative delays need to be taken into account. As set before, the sampling plan is composed of two parts. Once the sampling frame is defined, we first draw a sample to be interviewed-surveyed and then (after the fieldwork), we correct the possible non-response.

\subsection{Sampling plan}

Sampling consists on drawing a subset of units or elements from a finite population for the purpose of extrapolating the results obtained on this subset to the entire population. This selection can be probabilistic or deterministic. Deterministic samples are not random and are not used very often. They are mostly used to test the survey questionnaire. This type of design is known as quota sampling. Probabilistic samples are drawn from a population using a sampling scheme and sampling design.

In a probability sample, each unit in the population has a known selection probability. A probability method is used to select the specific units to be included in the sample. There are many sampling schemes, see for example the recent book of Tillé (2019), which contains a deep review on sampling methods. We briefly explain the ones most used at LISER:

1 Stratified random sampling. The population is partitioned in groups called strata, that are homogeneous within them but heterogeneous between them. Stratify random sampling might be used when the groups (the strata) are of the interest of the research, i.e. there is an interest to compare several groups. Also, when prior information is available for groups. Different costs within the strata are another of the reasons to stratify the population. On the other hand, when the strata show large variability for some variable being studied (Blair \& Blair, 2014), it is convenient to stratify the population so that the overall variance of the estimator is reduced, in fact Neyman (1934) proposed in these cases to obtain sizes proportional to the strata and to the variable under study for the strata.

2 Cluster sample. The observation units that compose a population are joined in sampling units of bigger size, the clusters. In clusters, the population is heterogeneous within the cluster and homogeneous between them. The book by Blair \& Blair (2014) contains the most common cases in which cluster sampling is used. Mainly the use of cluster sampling is associated to the cost of data collection and the fact of not having a list of all units of the population under study but the list of clusters. For instance, 
we can think a survey on children to be done by sampling schools and not directly kids.

3 Simple random sampling. It is used when auxiliary information is not available. In this case, it obtains a random sample of a fixed size. There are two forms, with and without replacement. The most used one is without replacement.

There is not a perfect design valid for all surveys. We should definitely take advantage of the auxiliary information to choose the accurate sampling scheme. One way can be including the auxiliary information into the sampling design (schema) through the stratification variable. If the variable of interest is positively correlated to the auxiliary variables and the variability increases with them, then the optimal stratification design may be one that takes into account larger sampling fractions in the strata with larger value of auxiliary variables and smaller sampling fraction in the strata with small value of auxiliary variables. That means that within stratified random sampling the most efficient sampling schema is not always necessarily the same allocation. That was already set by Neyman (1934). This idea is linked to the survey representativeness concept. According to the popular believe representativeness implies that the sample is a mirror image of the population and therefore representativity is assured by this simple relationship between sample and population. Quota sampling is often justified using this argument. Regarding quota sampling the concept of representativeness is falacious since there is a self-selection bias of the respondents, but also because of the interest to misrepresent some population categories in surveys. Another point of view is proposed by Davern (2008) that is also collected in the book from Tillé \& Matei (2016): When using a sample survey to make inferences about the population from which the sampled elements were drawn, researchers must judge whether the sample is actually representative of the study population. The best way to ensure it is a) have a complete list of all elements in the population b) ensure that each and every element on the list has nonzero probability of being included in the sample c) gather complete data from each and every sampled element. But let us think about that to think now, when there is non-response, where is the representativeness? how do you justify it? and furthermore, we are under the hypothesis of non-coverage problem. Is that realistic?

\subsection{Correction of non-response}

The procedure of non-response is run after the fieldwork data collection but is done at the level of the sample, meaning it is not corrected only for the respondents but for all people selected in the sample. But as set before, this procedure should be foreseen when designing the sampling plan. Since the information obtained may serve to correct for the non-response. In this subsection, we give a brief overview on the most important concepts for the non response focusing on the importance of having good information to adjust 
for non-response. Further details on non-response theory can be found on the monografy by Dillman et al. (2002). From the survey methodology point of view, most surveys typically have two types of errors, sampling errors and nonsampling errors. Sampling errors exist because we observe a set of the population and not the whole population. Non-sampling errors come from different sources such as frame imperfections and non-response.

1 Sampling error: Sampling error arises because we do not examine the whole population but a part of it. The sampling error derives from the random selection of units and it is therefore inherent to any type of sampling technique. Under the assumption of no other errors, the sampling error may be measured by the variance of the estimator in use, which is the sampling variance. The sampling variance might be affected by the variability of the target parameter; the population size; the sampling design; the sample size, knowing that when sample size increases, the variance decreases; the estimation method.

We may consider the Horowitz-Thompson (HT) estimator proposed by Horvitz \& Thompson (1952) when estimating a parameter. In the ideal case of a complete response (and no other source of error), we obtain the HT estimator variable of interest in the target population by adding up the weighted value of the variable of interest for each unit in the gross sample. Moreover, under this scenario, we can obtain an exact estimation of the total population by adding up the weights of the sample.

2 Non-sampling errors: We distinguish between two main errors: nonresponse and frame imperfections. Non-response may be divided into two different errors: non-contact and refusal.

a) Non-contact: This type of error occurs if no information at all can be collected about the status of that particular unit, i.e. not permitting to determine whether the unit is still part of the population of interest.

b) Refusal: The unit refuses to answer, meaning that no information can be collected about the variable of interest. Within this category we can dissociate between the complete non-response or partial (item) non-response.

In the context of frame imperfection, we distinguish between non-eligibility and duplication error.

a) Non-eligibility: The unit is not part of the population of interest (anymore), i.e. deceased, moved abroad or lives in a collective household (this last case depends on what is considered in the definition on the population, but persons living in collective institutions are often excluded from the sampling frame in household surveys in Luxembourg -HFCS and SILC-). 
b) Duplication error: Typically, the sampled unit corresponds to the unit of interest, meaning that weights obtained after the correction for nonresponse correspond to the final weights. For some surveys, it is not the case. That is the problem we have in HFCS: the sampled unit is a fiscal household while the unit of interest is the private household. The problem here is that a private household can be composed of several fiscal households e.g. a couple (private household) where both work and they are not married but have a civil partnership (pacte civil de solidarité - PACS) are considered in the IGSS as two separate fiscal households. This implies that we cannot always assume a one-to-one relationship between the fiscal household and the private household. In those cases there is a duplication of the units in the sampling frame, which means that we can select the same household more than once (in the example of the PACS, we can select both fiscal households and we will find the same private household). This situation may introduce a large bias in the estimators. After correcting the weights for nonresponse, we apply the Generalized Weight Share Method (GWSM) procedure as explained in Lavallée (2007) which helps us compute the correct weight of each private household.

These types of non-response are not always identifiable, and this is due in large measure to the survey mode. We often use two survey modes: face-to-face and web. It is easy to see that the only information we have in web surveys is the yes-no (complete or partial no) response, whereas on the face-to-face surveys, the non-response can be split by the types mentioned above (no because we did not contact the person, no because the person is not part of the population anymore, no because the unit refuses to answer). Non-response and frame imperfections may lead to large biases estimators. This makes the correction of each type or error (non-contact, refusal, non-eligibility and duplication) crucial for achieving unbiased population estimates. However, each error is treated in different ways, if there is no identification of the type of error, the bias of the estimator due to the non-response can be larger than expected.

On the other hand, the mechanism for the non-response (precisely, the noncontact and the refusal) may be the result of a random process. This random process can be completely random or uniform (in statistics is know as completely missing at random-CMAR). It also can be or random or ignorable (missing at random -MAR) when it depends on the auxiliary variables -that explain the variable of interest- which means that in order to correct for this type of nonresponse, we have to have a list of auxiliary variables that as mentioned above, should be available for all units in the sample. The third type is the nonresponse non-ignorable (not missing at random -NMAR) depends on the variable of interest, and it cannot be easily corrected.

Furthermore, the correction for the non-response implies estimators with lower efficiency (large variance). This is due to two main reasons: a reduction of the sample size and an increase in the variability of the weights. The weighting 
system is always a compromise between reducing the bias (as much as possible) and increasing the variance. In Figure 1, we plot for illustration the weighting procedure for the Household finance consumption survey (HFCS). The weighting sequence of Figure 1 is the following: First step is the correction for selecting a set of the population. In a second step, we lost part of the sample due to non-contact (for whatsoever reason). Then, the units that remain $\left(n_{c}\right)$ should "compensate" the lost units $\left(n_{n c}\right)$. Within contacted units $n_{c}$ there are units that are not part of the population of interest anymore, they are called non-elegilible units $\left(n_{n e}\right)$. Non-elegilible units should be removed also from the population. After this correction, we are left with the elegilible units $\left(n_{e}\right)$. Next, not all elegilible units agreed to answer. The respondents $\left(n_{r}\right)$ should "compensate" for the no-respondents $\left(n_{n r}\right)$. In then HFCS, we have also to account for the problem of duplication units, in which, for some cases, we select the same unit more than once. The study units $\left(n_{s}\right)$ may "compensate" for this problem.

Aside from sampling and non-sampling error, a major source of error in surveys is the measurement process. A big component of measurement is the questionnaire and the process that respondents use to understand a question and retrieve relevant information, how they respond given the response options, and how they communicate this response (Biemer \& Lyberg, 2003). There can be many obstacles between the respondent and the response, and we will focus more thoroughly on this problem in a forthcoming document on questionnaire design.

\section{Estimation of the parameters}

As we point out throughout the whole document, the aim of the survey is the estimation of a characteristic of the population or the parameter. The estimator has always attached a measure of efficiency: the variance. The estimation of the parameter and the associated variance depend on the proposed sampling plan and also on the correction of non-response. The book of Tillé (2019) contains the computation of the estimators for means, totals an complex parameters such as quantities. Moreover this book contains a chapter on estimation of the variance via linearization. Typically, the estimation of the variance of the estimator does not take into account the correction of non-response. Under non-response, the analytical computation of the variance becomes quite complicated then empirical methods are used. The report from Bienvenue (2001) contains the estimation of the variance under the presence of non-response by assuming the non-response as sub-sample of the sample obtained by Poisson sampling. Another option is to implement the bootstrap method of Rao et al. (1992), which can be used under the stratified random sampling.

Typically the estimators are used take into account the weights obtained after the adjustment for non-response. The weighting system is the end of the whole process. The weighting system is the tool that allows to infer the parameters of the population with the data from the (sample) of respondents. 
Figure 1: Weighting procedure in the LU-HFCS.

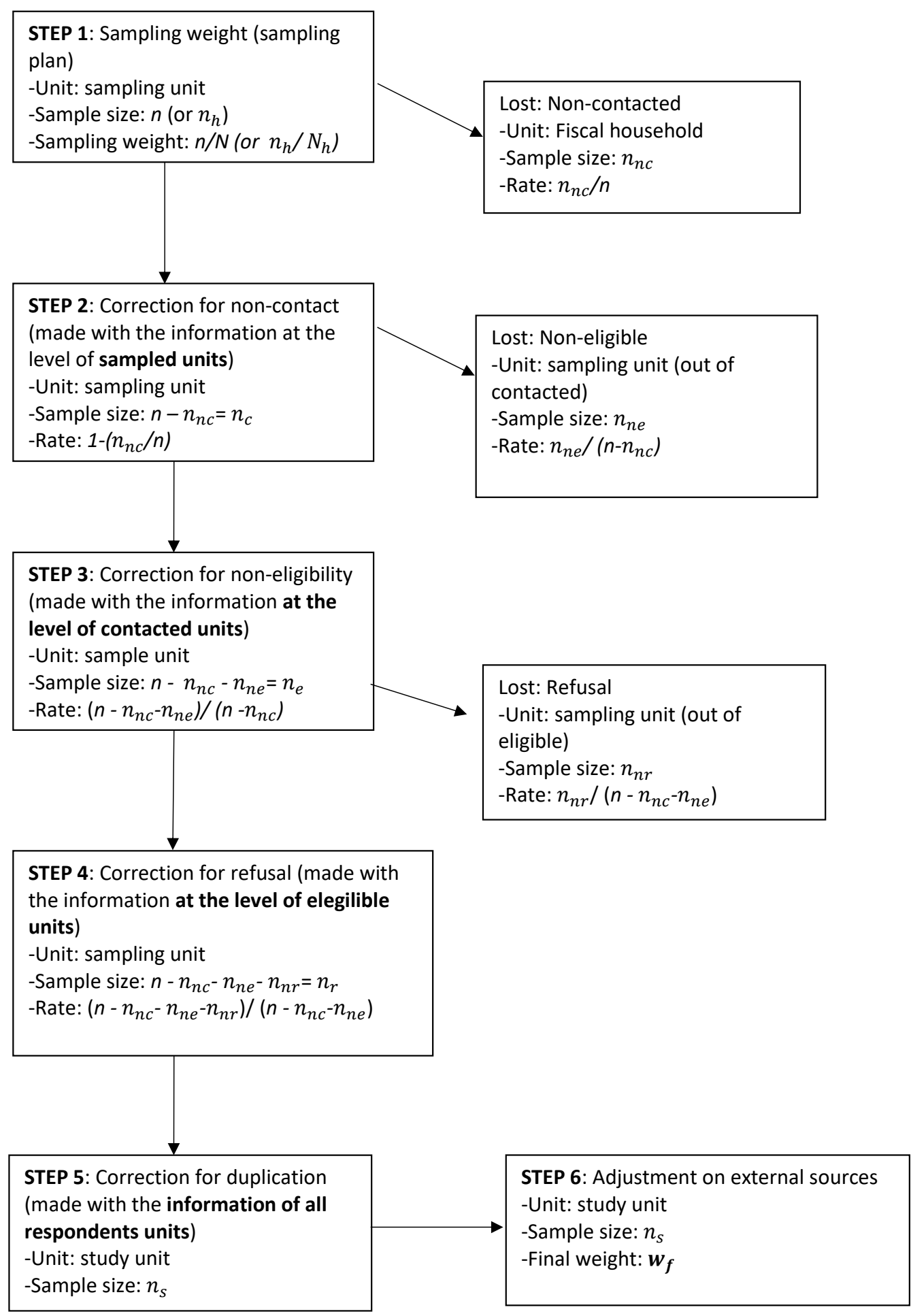


The results obtained by applying the weighting system (in form of weighted estimators) should be then coherent. That means that the weighting system is made regarding the variable of interest taking into account the whole procedure (sampling, fieldwork and correction for non-response). The weighting system is done always on the final data base coming from the fieldwork, where final here means the correctly cleaned data base (by considering and analyzing the possible errors in the data base coming from the fieldwork). The weighting system will be valid if the results obtained with the weighted estimator are coherent.

\section{Acknowledgments}

The authors would like to deeply thank the careful read of professor Yves Tillé and his valuable comments. The authors are also thankful for the suggestions of their colleges from DC department Jean-Yves Bienvenue, Carla Martins and Thierry Kruten.

\section{References}

Biemer, P. P. \& Lyberg, L. E. (2003). Introduction to survey quality, vol. 335. John Wiley \& Sons.

Bienvenue, J.-Y. (2001). Redressement d'un échantillon en une phase par calage sur marges. Document de travail ms2001-1, CEPS/Instead, Luxembourg.

Blair, E. \& Blair, J. (2014). Applied survey sampling. Sage Publications.

Brancato, G., Macchia, S., Murgia, M., Signore, M., Simeoni, G., Blanke, K. \& Hoffmeyer-Zlotnik, J. (2006). Handbook of recommended practices for questionnaire development and testing in the european statistical system. European Statistical System .

Bryman, A. (2016). Social research methods. Oxford university press.

Burgess, T. F. (2001). A general introduction to the design of questionnaires for survey research. UK: University of Leeds .

DAVERn, M. (2008). Representative sample. Encyclopedia of survey research methods , 721-723.

De Vaus, D. (2002). Surveys in social research . crows nest. New South Wales: Allen and Unwin .

Dillman, D. A., Eltinge, J. L., Groves, R. M. \& Little, R. J. A. (2002). Survey nonresponse. 
Fowler, F. J. \& Cosenza, C. (2008). Writing effective questions. International handbook of survey methodology 8, 136-159.

Franklin, S. \& Walker, C. (2003). Survey methods and practices. statistics canada. Social Survey Methods Division, Ottawa .

Hartley, H. O. (1974). Multiple frame methodology and selected applications. Sankhya 36, 118.

Horvitz, D. G. \& Thompson, D. J. (1952). A generalization of sampling without replacement from a finite universe. Journal of the American Statistical Association 47, 663-685.

IGSS (2019). Luxembourg Microdata Platform on Labour and Social Protection. Data Dictionary. Tech. rep., Inspection générale de la sécurité sociale.

JAnn, B. \& Hinz, T. (2016). Research question and design for survey research. In Wolf et al. (2016), pp. 105-121.

LavallÉE, P. (2007). Indirect sampling. Springer Science \& Business Media.

LoHR, S. (2007). Recent developments in multiple frame surveys. cell 46, 6 .

LoHr, S. L. (2009). Multiple-frame surveys. In Handbook of statistics, vol. 29. Elsevier, pp. 71-88.

LoHr, S. L. (2010). Sampling: Design and analysis (2nd ed.). Brooks/Cole: Boston, USA.

Lyberg, L. \& Weisberg, H. (2016). Total survey error: a paradigm for survey methodology. In Wolf et al. (2016), pp. 27-41.

NeYman, J. (1934). On the two different aspects of the representative method: the method of stratified sampling and the method of purposive selection. Journal of the Royal Statistical Society 97, 558-625.

RaO, J., Wu, C. \& Yue, K. (1992). Some recent work on resampling methods for complex surveys. Survey methodology 18, 209-217.

Schwarz, N. (1997). Questionnaire design: The rocky road from concepts to answers. Survey measurement and process quality , 27-45.

STATEC (2018). Répertoire des entreprises luxembourgeoises. Tech. rep., Institut national de la statistique et des études économiques.

Tillé, Y. (2019). Théorie des sondages-2e éd.: Échantillonnage et estimation en populations finies. Cours et exercices corrigés. Dunod, Paris.

Tillé, Y. \& MAtei, A. (2016). Basics of sampling for survey research. In Wolf et al. (2016), pp. 311-328. 
Wolf, C., Joye, D., Smith, T. W. \& Fu, Y.-C. (2016). The SAGE handbook of survey methodology. London, London, UK: Sage. 


